\title{
Muscle reorganization following incomplete cervical spinal cord injury in rats
}

\author{
M Nakamura, Y Fujimura, Y Yato and M Watanabe \\ Department of Orthopaedic Surgery, School of Medicine, Keio University, 35 Shinanomachi, Shinjuku-ku, Tokyo, \\ 160, Japan
}

\begin{abstract}
This study on rats was designed to evaluate the change of biceps muscle fibers by enzyme histochemical examination and the change of distribution of motoneurons innervating biceps muscle fibers by retrograde tracer examination after incomplete spinal cord injury. Incomplete spinal cord injury was produced by placing a $20 \mathrm{~g}$ weight on exposed dura at the C6 level. The number of the labeled motoneurons of C6 segment significantly decreased compared with that in the control, but there were no significant changes in the other segments at 4 weeks after the injury. Moreover, there was type grouping of biceps muscle fibers at 4 weeks after the injury. These findings indicated that the incomplete cervical spinal cord injury at C6 level in rats caused the partial denervation and then reinnervation of biceps muscle fibers by the collateral sprouting of the remaining motoneurons which belonged to the same motoneuron pool of the injured motoneurons.
\end{abstract}

Keywords: incomplete cervical spinal cord injury; partial denervation; motoneuron; muscle fiber type grouping

\section{Introduction}

It is well known that a patient with incomplete cervical spinal cord injury (SCI) can recover to a varying degree, depending on the severity and extent of the initial spinal cord injury. ${ }^{1}$ Hopkins and Rudge have tried to prove Schneider's explanation about the relative sparing of the legs in incomplete cervical SCI, but there was no evidence of lamination of the pyramidal tract in the human. ${ }^{2}$ Functional recovery following incomplete cervical SCI has not been fully explained so far. One of the reasons of this is that the functional changes of the gray matter, especially of the spinal motoneurons, have not yet been clarified despite of the fact that central gray matter damage was a common finding of incomplete SCI. In our previous report, we demonstrated that rat motor function fully recovered at 4 weeks after incomplete cervical SCI, despite of the irreversible changes in the motoneurons at the injured site and concluded that it might be due to the compensatory reaction of the motoneurons neighboring to the injured site. $^{3}$ We hypothesized that this compensatory reaction caused the reinnervation of partial denervated muscle fibers by the peripheral sprouting of the neighboring motoneurons which belonged to the same motor neuron pool of the injured motoneurons. In order to clarify this hypoth-

Correspondence: M Nakamura esis, this study was designed to evaluate the change of biceps muscle fibers by enzyme histochemical examination and the change of distribution of motoneurons innervating biceps muscle fibers by retrograde tracer examination after incomplete SCI.

\section{Materials and methods}

Twelve male Wistar rats, weighing 250 to $280 \mathrm{~g}$, were used in this study. The rats were provided by Nippon Bio-sup. Center (Tokyo, Japan), and were housed at room temperature $\left(22 \pm 2.4^{\circ} \mathrm{C}\right)$ and exposed to a $12 \mathrm{~h}$ light/dark schedule $(6.00 \mathrm{am}-6.00 \mathrm{pm})$ for more than 1 week before use in the experiments.

\section{Incomplete cervical spinal cord injury model}

Each rat was anesthetized with intraperitoneal pentobarbital $(40 \mathrm{mg} / \mathrm{kg})$. Incomplete cervical SCI model was produced by the previous method. ${ }^{3}$ In brief, a laminectomy was performed at the level of $\mathrm{C} 5$ and C6. The cervical spinal column was stabilized by fixing the skull and the spinous process of T2. The base-plate $(2.2 \times 3.0 \mathrm{~mm})$, with a $20 \mathrm{~g}$ weight, was centered over the laminectomy site and placed onto the exposed dura at the C6 level for $5 \mathrm{~min}$ (injury group: $n=6$ ). Only a laminectomy was performed without compression at the same level in control $(n=6)$. 


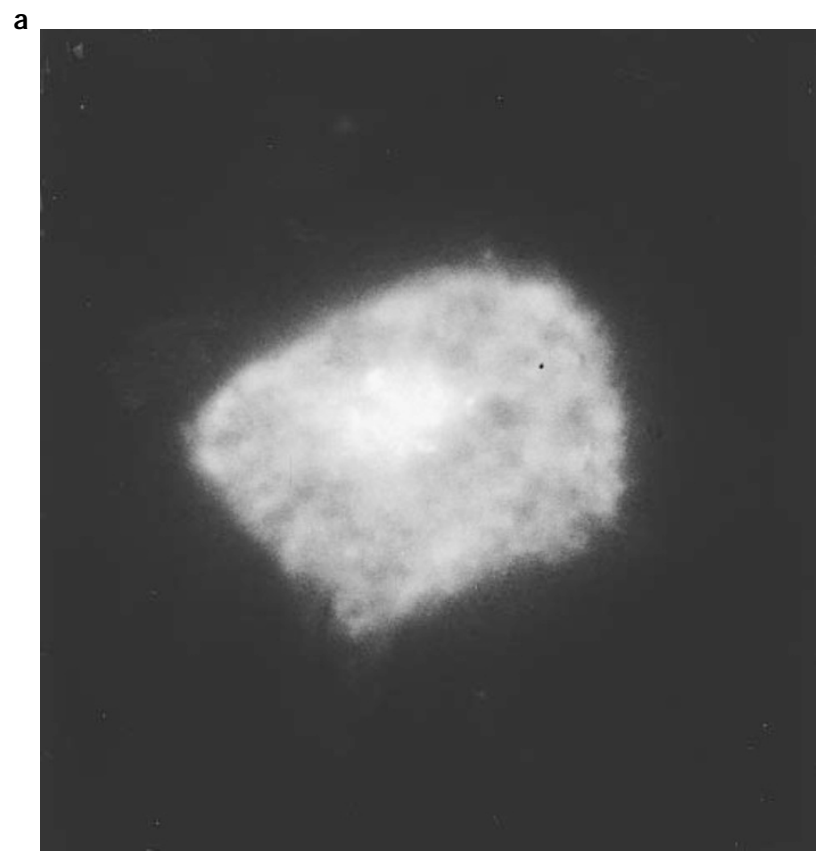

b

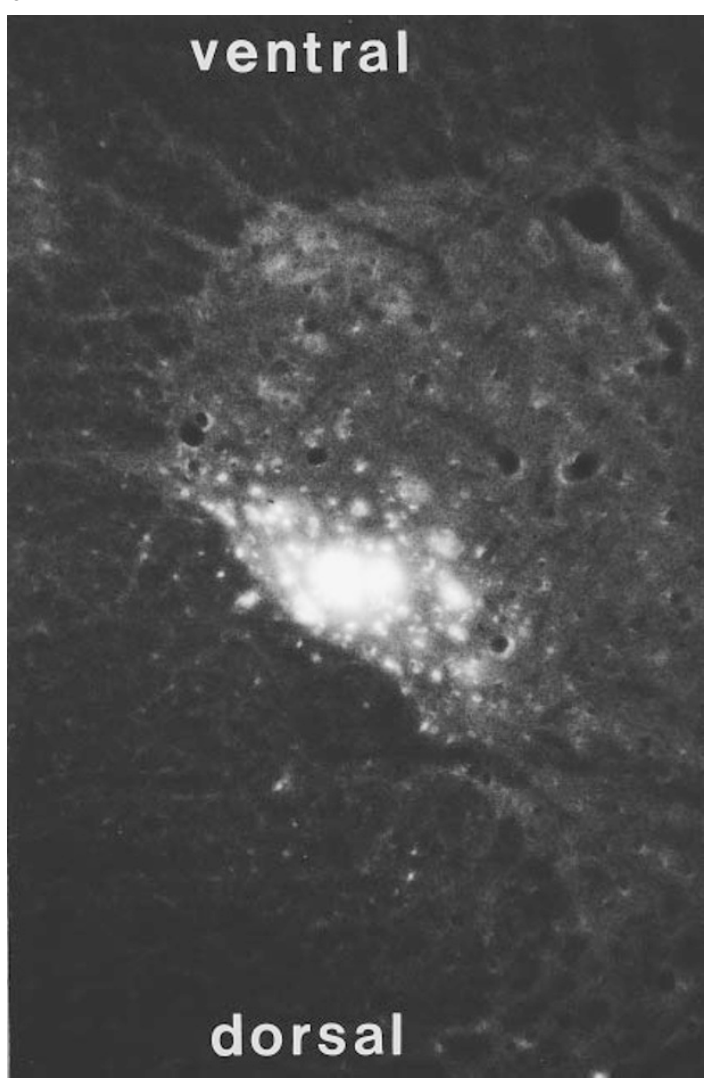

C

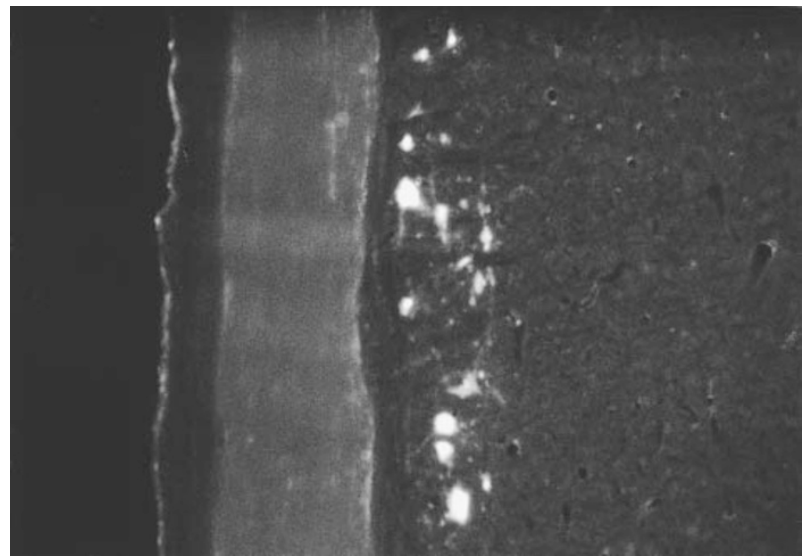

Figure 1 Fluorescence photomicrographs to show that bisbenzimide can be transported from the biceps muscle to motoneurons in cervical spinal cord. (a) A large retrogradely labeled motoneuron which was typically stained by bisbenzimide at $24 \mathrm{~h}$ after the injection. Bisbenzimide-labeled motoneurons were distributed in the spinal cord from $\mathrm{C} 4$ to $\mathrm{C} 7$ segments, mainly in C5 and C6 segments along a column located at the posterolateral aspect of the ventral horn. (b) Transverse section, (c) coronal section in $\mathrm{C} 5$ segment of the spinal cord 
Retrograde labeling with fluorescent dyes

Bisbenzimide (Shigma) was used as a $10 \%$ aqueous solution. Injections were made using conventional methods. We employed a $50 \mu \mathrm{l}$ Hamilton syringe equipped with a microdrive. In six rats (injury group: three, control: three), $50 \mu$ l bisbenzimide was injected into biceps muscle at 4 weeks after the operation. At $24 \mathrm{~h}$ after the injection, transcardial perfusion with $4 \%$ paraformaldehyde ( $\mathrm{pH}$ 5.6) was performed. Cervical spinal cord from segments $\mathrm{C} 4$ to $\mathrm{C} 7$ was removed and frozen in isopentane cooled in liquid nitrogen. Serial horizontal sections, $20 \mu \mathrm{m}$ thick, of the spinal cord were cut in a cryostat at $-20^{\circ} \mathrm{C}$. Motoneurons were identified using a fluorescence microscope (Nikon, Tokyo) at $360 \mathrm{~nm}$ wave length and all the labeled motoneurons in which the nucleus was clearly visible were counted in 20 sections. Difference in the number of the labeled motoneurons between the injury group and control was compared using Mann-Whitney Utest. Statistical significance was defined as $P<0.05$.

\section{Enzyme histochemical examination}

Biceps muscle was removed at 4 weeks after the operation in the other six rats (injury group: three, control: three). Fresh biceps muscle tissue was flash frozen in isopentane, and cooled to $-160^{\circ} \mathrm{C}$ using liquid nitrogen and then sectioned at $10 \mu \mathrm{m}$ in a cryostat. Enzyme histochemistry for myofibrillar adenosine triphosphatase (ATPase) activity was conducted on the sections according to the method of Brooke et al. The $\mathrm{pH}$ during preincubation of muscle fibers in ATPase reactions were $\mathrm{pH} 10.4,4.60$ and $4.33{ }^{4}$ The distribution pattern of muscle fibers was examined under the microscope level.

\section{Results}

Retrograde neuronal labeling

In the control, bisbenzimide-labeled motoneurons were distributed in the spinal cord from $\mathrm{C} 4$ to $\mathrm{C} 7$ segments, mainly in C5 and C6 segments along a column located at the posterolateral aspect of the ventral horn (Figure 1a, b, c). In injury group, the number of the labeled motoneurons of C6 segment significantly decreased compared with that in the control. On the other hand, there were no significant differences in the numbers of the labeled motoneurons of C4, C5 and C7 segments between the injury group and control (Figure 2).

\section{Biceps muscle fiber type distribution}

In the control, type 1 fibers stained light at $\mathrm{pH} 10.4$ and dark at $\mathrm{pH} 4.60$ and 4.33. Type 2A fibers stained dark at $\mathrm{pH} 10.4$ and light at $\mathrm{pH} 4.60$ and 4.33. Type 2B fibers stained dark at $\mathrm{pH} 10.6$ and 4.60 and light at $\mathrm{pH}$ 4.33. There was no grouping of muscle fiber type in Control (Figure 3a). In the injury group, biceps muscles showed a marked histochemical reorganization with prominent fiber-type grouping as shown by ATPase reaction at 4 weeks after the incomplete SCI (Figure 3b).

\section{Discussion}

The grouping of muscle fibers is thought to be related to the innervation patterns of the motoneurons which reinnervate the muscle and/or to collateral sprouting

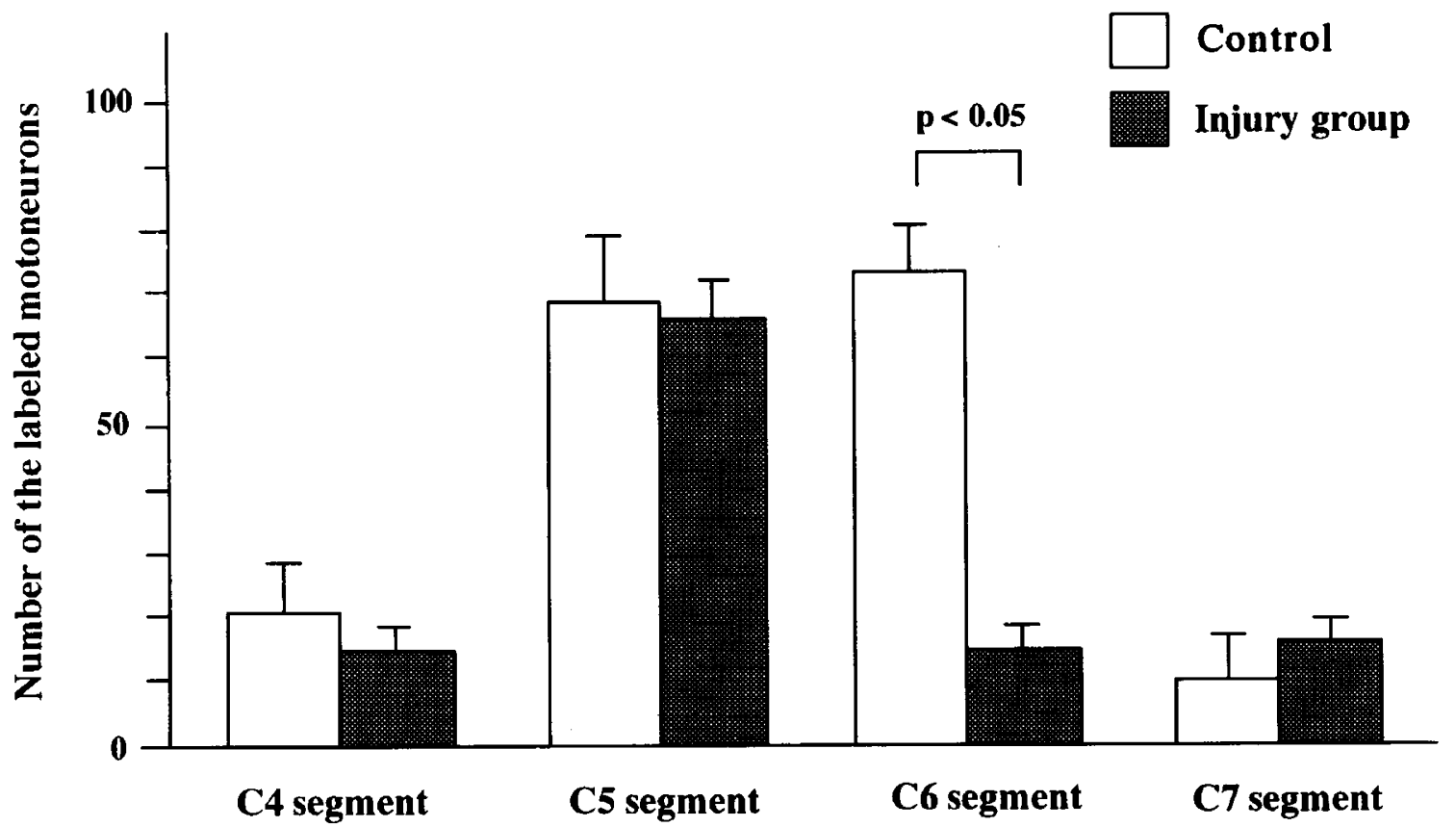

Figure 2 Comparison of the number of labeled motoneurons of each segment between the control and injury group 
a

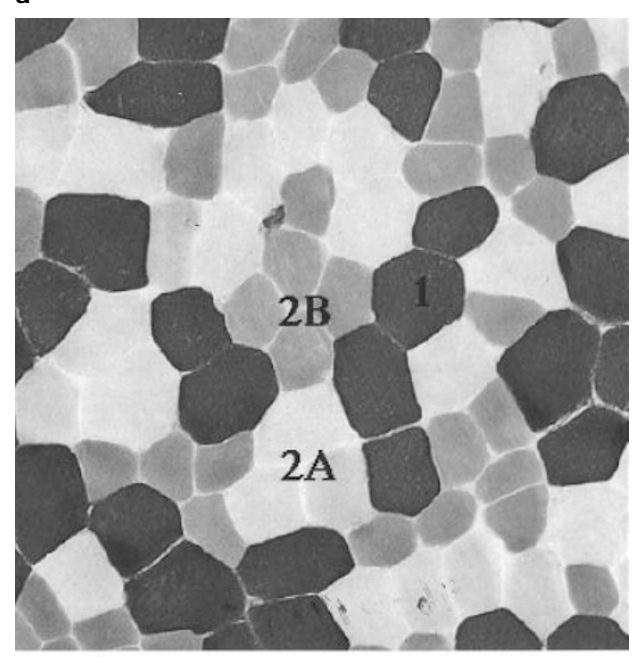

b

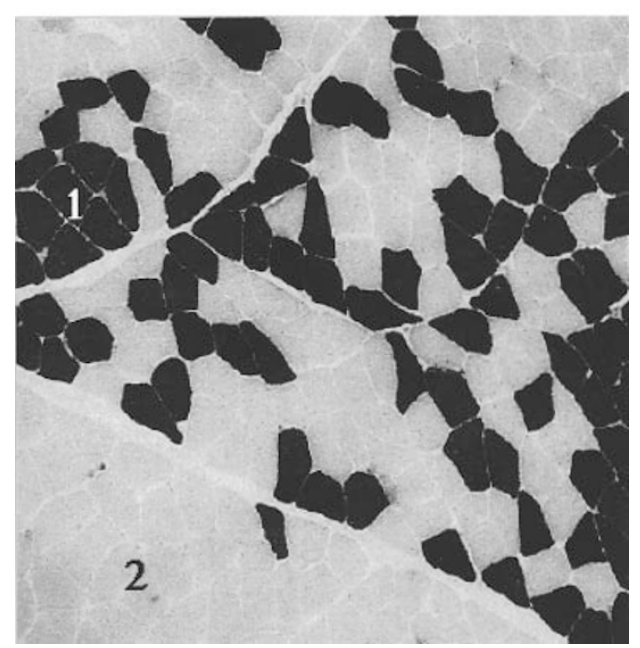

Figure 3 (a) Cross section of biceps muscle in control (ATPase reaction, $\mathrm{pH} 4.60$ ). Biceps muscle consisted of type 1 , type $2 \mathrm{~A}$ and type $2 \mathrm{~B}$ fibers and these fibers showed a mosaic pattern. (b) Four weeks after the incomplete cervical spinal cord injury, biceps muscle fibers showed a intense fiber-type grouping (ATPase reaction, $\mathrm{pH} 4.33$ )

from existing innervated neuromuscular endplates. The absence of 'type grouping' has often been used to indicate the absence of denervation and reinnervation. ${ }^{5}$ We demonstrated that biceps muscle showed a marked histochemical reorganization with prominent fiber-type grouping at 4 weeks after the incomplete cervical spinal cord injury at C6 level. This finding indicated that the incomplete cervical spinal cord injury at C6 level in rats caused the partial denervation and reinnervation of biceps muscle fibers. Moreover, in our retrograde tracer experiment, the motoneurons innervating the control biceps muscle fibers were distributed at the spinal cord from $\mathrm{C} 4$ to $\mathrm{C} 7$ segment. On the other hand,
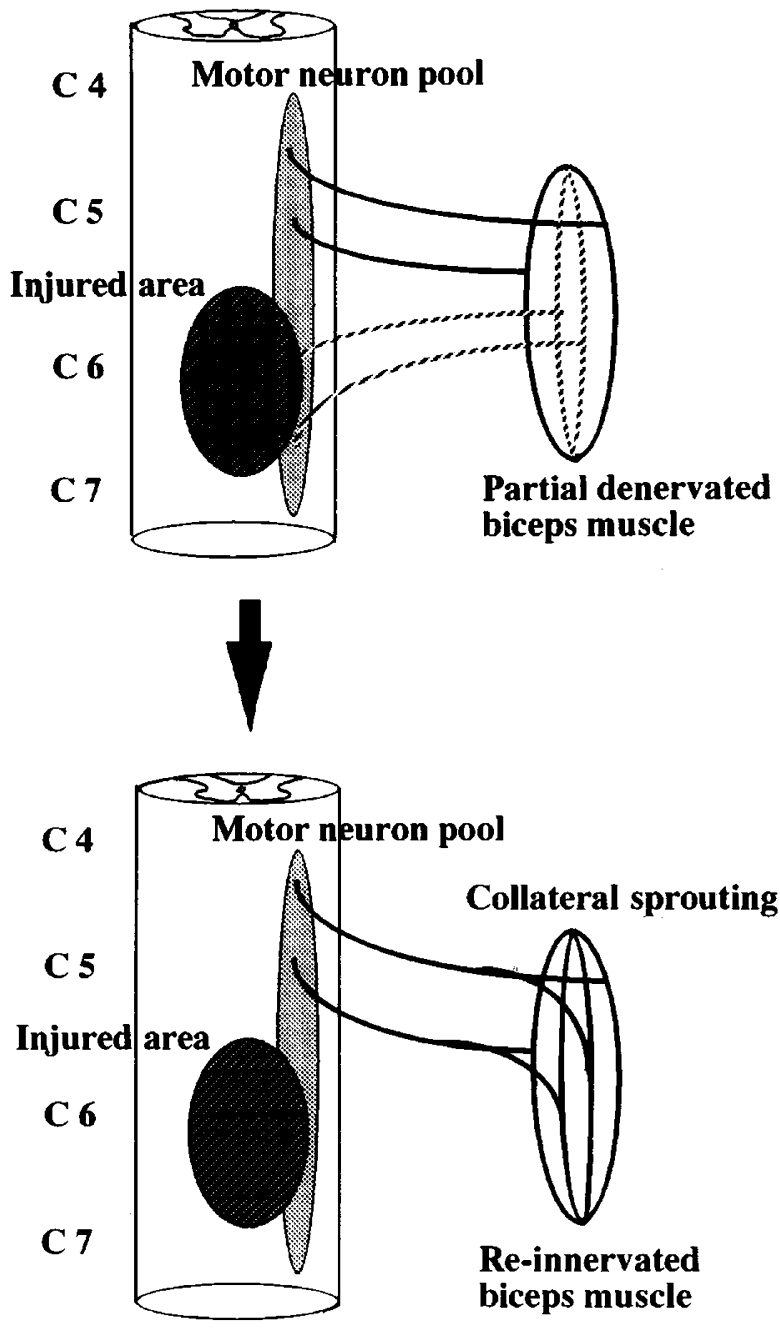

Figure 4 Our hypothesis. Muscle reorganization after an incomplete cervical spinal cord injury

at 4 weeks after the incomplete cervical spinal cord injury at C6 level, the motoneurons innervating the biceps muscle fibers were distributed mainly at C5 segment and the number of the labeled motoneurons at C6 segment significantly decreased compared with the control. These findings indicated that the partial denervated biceps muscle fibers were reinnervated by the collateral sprouting of the remaining motoneurons which belonged to the same motoneuron pool of the injured motoneurons (Figure 4).

Moreover, these findings accorded with the results of our previous study that the incomplete cervical spinal cord injury resulted in a persistent low level of ChAT activity in the compressed spinal cord segment and late over-expression of ChAT activity were found in the spinal cord segments located both rostral and caudal to the injury. ${ }^{6}$ This phenomenon is probably what is called muscle reorganization following an incomplete cervical spinal cord injury. 


\section{References}

1 Bohlman $\mathrm{HH}$ et al. Mechanical factors affecting recovery from incomplete cervical spinal cord injury: A preliminary report. John Hopkins Med J 1979; 145: $115-125$

2 Hopkins A, Rudge P. Hyperpathia in the central cervical cord syndrome. J Neurol Neurosurg Psychiatr 1973; 36: 637-642.

3 Nakamura $\mathrm{M}$ et al. Changes in choline acetyltransferase distribution in the cervical spinal cord after reversible cervical spinal cord injury. Paraplegia 1994; 32: $752-758$.

4 Brooke R, Kaiser KK. Muscle fiber types: How many and what kind? Arch Neurol 1970; 23: 369 - 379.

5 Sue $\mathrm{C}$ et al. Innervation patterns in the cat tibialis anterior six months after self-reinnervation. Muscle Nerve 1993; 16: 379391.

6 M Nakamura et al. Changes in choline acetyltransferase activity and distribution following incomplete cervical spinal cord injury in the rat. Neuroscience 1996; in press 